\title{
Uma estratégia de Game Learning Analytics para avaliar level design em um jogo educacional
}

\author{
Douglas Silva $^{1}$, Rafaela Melo ${ }^{1}$, João Bernardo ${ }^{1}$, Marcela Pessoa ${ }^{1}$, Luis Cuevas \\ Rodríguez $^{1}$, Fernanda Pires ${ }^{1}$ \\ ${ }^{1}$ Escola Superior de Tecnologia - Universidade do Estado do Amazonas (UEA) \\ ThinkTEd Lab - Pesquisa e Desenvolvimento em Tecnologias Educacionais \\ Manaus - AM - Brasil \\ \{dsm.lic17,rmf.lic16,jrsbr.lic16,msppessoa, lrodriguez,fpires\}@uea.edu.br
}

\begin{abstract}
This paper aims to evaluate the level design of an educational game called "Robô Euroi" using Game Learning Analytics (GLA) techniques, with its own flexible data capture structure. An application of the game was run with 71 people of different ages, where the data generated by the players were captured and later analyzed using the Python programming language. The results show the benefits of capturing and analyzing applied data, good acceptance by the target audience, and a challenging potential for the game.
\end{abstract}

Resumo. Este trabalho tem como objetivo avaliar o level design de um jogo educacional chamado "Robô Euroi" usando técnicas de Game Learning Analytics (GLA), com uma estrutura própria de captura de dados flexível. Uma aplicação do jogo foi realizada com 71 pessoas de diferentes idades e os dados gerados pelos jogadores foram capturados e posteriormente analisados, usando a linguagem de programação Python. Os resultados evidenciam benefícios da captura e análise de dados aplicadas, uma boa aceitação por parte do públicoalvo e um potencial desafiador do jogo.

\section{Introdução}

Pesquisas defendem o potencial da utilização de jogos sérios educacionais no processo de aprendizagem, através do desenvolvimento de habilidades e da solução de problemas, mas, entender como isso acontece ainda é um desafio [Kang et al. 2017]. Geralmente, os pesquisadores fazem uso de testes heurísticos para avaliar seus jogos. Porém, os jogos são compostos por elementos que, na maioria das vezes, não são explorados nessas avaliações, mas que podem contribuir para análises mais robustas de aprendizagem.

Os dados gerados a partir da interação do usuário com o jogo, podem fornecer informações úteis sobre o processo de aprendizagem do jogador [Freire et al. 2016]. Esses dados também podem apontar para erros de game e level design. Com a Game Learning Analytics (GLA) [Freire et al. 2016] é possível analisar tais dados sem a necessidade de interromper o jogador, evitando interferir no desempenho ou causar uma sensação de desconforto. Quando se trata de jogos comerciais, a análise de dados está relacionada a melhorar a experiência do usuário, encontrar erros e apoiar o crescimento dos jogos [Freire et al. 2016]. Geralmente são utilizados serviços externos para realizar as análises, visto que, já existe um mercado nesta área de atuação. Para jogos educacionais, além dos elementos de entretenimento, existem os traços de aprendizagem. É complexo analisar os 
dados, uma vez que os elementos de aprendizagem estão incorporados aos elementos de mecânica [Van Eck 2006].

Os dados coletados em jogos educacionais permitem fazer diversos tipos de análise. É possível verificar perfis de usuário, prever o desempenho, adaptar o jogo, analisar o game design, replicar a trajetória do jogador, comparar comportamentos, investigar dificuldades [Alonso-Fernández et al. 2019a]. A partir dessas análises, os desenvolvedores e pesquisadores podem ajustar os seus jogos e conferir o desempenho dos alunos.

O presente trabalho tem por objetivo propor um processo para captura e análise de dados oriundos da utilização do Robô Euroi [Melo et al. 2018a], um jogo educacional que tem como proposta promover o desenvolvimento do Pensamento Computacional através da promoção de aprendizagem em Matemática. Através das análises realizadas nesta pesquisa, foi possível identificar pontos de dificuldade encontrados pelo jogador e verificar se são problemas causados pelo level design elaborado ou falhas do próprio usuário.

A sequência do artigo está estruturada da seguinte forma: a Seção 2 discorre sobre os jogos educacionais e a análise de aprendizagem; na Seção 3 são apresentadas as especificações do jogo; a Seção 4 apresenta o design experimental; nas seções 5 e 6 estão os resultados e as conclusões, respectivamente.

\section{Jogos educacionais e análise de aprendizagem}

Serious game (jogo sério) é o termo atribuído a jogos digitais que têm objetivos além do entretenimento, podem ser: simulações de treinamento, política, economia, aspectos sociais e por vezes são classificados de educacionais [Djaouti et al. 2011]. Jogos sérios educacionais são caracterizados por envolver atividades através de narrativa e jogabilidade para transmitir algum significado ao jogador e que faça parte do currículo escolar [Plass et al. 2015].

Embora os jogos sérios venham sendo investigados como possíveis beneficiadores do processo de aprendizagem, ainda há a necessidade de entender como isso pode ocorrer, em função disso, é importante que passem por avaliações. Em alguns trabalhos os jogos são avaliados através de pré e pós-testes [Girard et al. 2013], análise por observação e outros tipos de questionário em papel [Kang et al. 2017]. No entanto, o ambiente de um jogo oferece ao jogador diferentes estratégias e caminhos para que possam alcançar o seu objetivo, e este percurso adquirido pelo jogador durante o jogo têm chamado a atenção de pesquisadores, visto que pode fornecer uma avaliação mais detalhada de como pode ocorrer a aprendizagem [Wallner and Kriglstein 2013].

A análise de aprendizagem em jogos (Game Learning Analytics - GLA) referese à extração, coleta e análise de dados fornecidos pelas interações de jogadores com jogos sérios [Freire et al. 2016, Alonso-Fernández et al. 2019a], a partir de dados como registro de data e hora, identificador do usuário, status de conclusão, pontuação, número de tentativas e demais eventos gerados por um jogador. Cada jogo educacional tem um objetivo específico de aprendizagem e possui estruturas diferentes, o que os faz gerar dados distintos, por isso, é difícil padronizar a coleta de dados para este tipo de jogo.

Através dos dados coletados em jogos, pode ser possível avaliar a aprendizagem, traçar perfis de estudantes, verificar padrões de comportamento e avaliar o game design desenvolvido. Nesse sentido, pesquisas em jogos sérios educacionais têm realizado 
avaliação por meio de técnicas de GLA, buscando entender o processo de aprendizagem do jogador. Alguns trabalhos que utilizam técnicas para identificar falhas no design do jogo ou outros aspectos são citados a seguir.

Em Hicks et al. (2016), padrões de jogabilidade são explorados para ajudar a entender o motivo de desistência de usuários dentro de um jogo sobre conceitos de física, chamado Quantum Spectre. Para realizar essa análise, os autores coletaram dados de jogabilidade de 433 alunos, divididos em dois grupos, um que jogou em sala de aula e outro fora desse ambiente. Os resultados obtidos por meio de análise de sobrevivência e análise de rede de interação apresentaram pontos problemáticos na progressão do nível em que os jogadores desistiram, além de diferenças potenciais entre os grupos analisados.

Kang et al. (2017) utilizaram dados do usuário em um jogo sobre ciências, intitulado Alien Rescue, para identificar padrões de comportamento na solução de problemas e explorar essas características para comparar grupos de desempenho. Os autores registraram as ações do jogo conforme o aluno interagia com o ambiente e organizava esses dados em uma tabela. Através das análises estatísticas realizadas, foram identificados cinco estágios de aprendizagem durante a aplicação e os alunos foram separados em grupos de alto e baixo desempenho.

Em Alonso-Fernández et al. (2019b), os autores aplicam GLA em três jogos sérios diferentes e com objetivos distintos, para validar e implantar um jogo de conscientização do cyberbullying, para validar o design de um jogo voltado para pessoas com deficiência intelectual e para melhorar a avaliação de um jogo sobre primeiros socorros. Na coleta de dados foi utilizada a ferramenta Experience API. A partir das aplicações realizadas com três grupos diferentes de alunos, os autores obtiveram resultados significativos com relação ao uso de GLA, puderam verificar se as opções de design se adequavam aos objetivos traçados e prever resultados de aprendizado que geralmente são medidos por questionários, o que simplifica a avaliação e a aplicação.

Assim como os estudos discutidos nesta seção, este artigo trata da avaliação de um jogo sério para a aprendizagem de matemática, através da coleta e análise de dados. Para coleta e armazenamento foi utilizado o recurso JSON e a análise está focada em verificar as áreas de dificuldade, a progressão dos níveis e o motivo da falha em alcançar o objetivo das fases (podendo ser mecânica ou lógica).

\section{O jogo Robô Euroi}

Robô Euroi ${ }^{1}$ é um jogo de ação e estratégia que aplica fundamentos matemáticos de forma implícita a fim de desenvolver o Pensamento Computacional. A mecânica do jogo é responsável pelos elementos de aprendizagem em matemática, o ato de ganhar e perder energia, ao pular entre plataformas, durante as fases refere-se à adição (ganhar energia) e à subtração (perder energia) e, para atingir a vitória, o jogador deve calcular bem as suas jogadas. Em trabalhos anteriores [Melo et al. 2018a, Melo et al. 2018b], foi apresentada uma versão do jogo implementada no Construct. A versão atual passou por modificações no level design e na composição e organização dos elementos gráficos, além disso, os desenvolvedores migraram para a ferramenta Unity, como descrito a seguir.

\footnotetext{
${ }^{1}$ Disponível em: https://drive.google.com/file/d/1uF9LJc6lhGsGDYxaw94jIZ977EdEWJM5/view?usp $=$ sharing
} 


\subsection{Especificações da interface}

Motivar o jogador através de elementos gráficos que compõem a interface do jogo foi um dos princípios utilizados no desenvolvimento do Robô Euroi. Além de constituírem a identidade visual do jogo, os elementos utilizados no design atuam como motivadores para criatividade e idealização de estratégias, destacando os aspectos mais importantes em cada tela, através da combinação de tipografias estilizadas com cores quentes. O padrão utilizado na organização, agrupamento e localização dos elementos contidos na interface do jogo, visam facilitar o processamento cognitivo do jogador, promovendo a análise das informações de forma clara e objetiva [Pires et al. 2020].

Como pode ser observado na Figura 1, o menu inicial e as demais telas do jogo fazem uso de elementos gráficos que permitem a navegação no sistema, possibilitando que o jogador transite entre as telas, acessando e alterando as configurações e seções disponibilizadas. Para auxiliar na captura de dados referentes ao perfil do usuário, o sistema disponibiliza um conjunto de avatares, que permite ao jogador identificar o gênero e a faixa etária de acordo com o seu perfil.

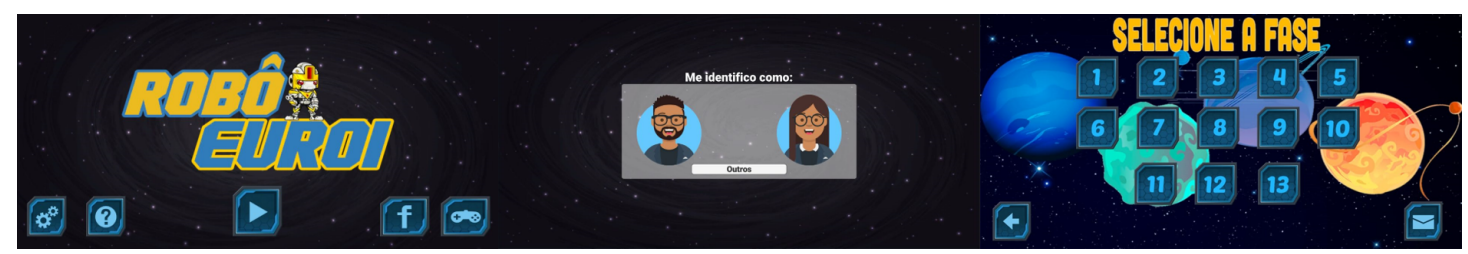

Figura 1. Telas do jogo: tela inicial, escolha do avatar e seleção de uma das treze fases do jogo.

No jogo, o personagem Robô Euroi é encaminhado para a missão de recuperar blocos de energia que foram perdidos em um planeta vizinho. Em meio a sua jornada, ele encontra diferentes tipos de plataformas: fixas, móveis, e aquelas que podem consumir entre uma e sete cargas de energia, como indicadas na Figura 2. Em cada um dos desafios enfrentados pelo jogador, existe um mapeamento específico de plataformas, este é matematicamente organizado e gerenciado por um algoritmo de acompanhamento de resultados, que posteriormente auxilia na análise de ritmo do jogador (Figura 2).

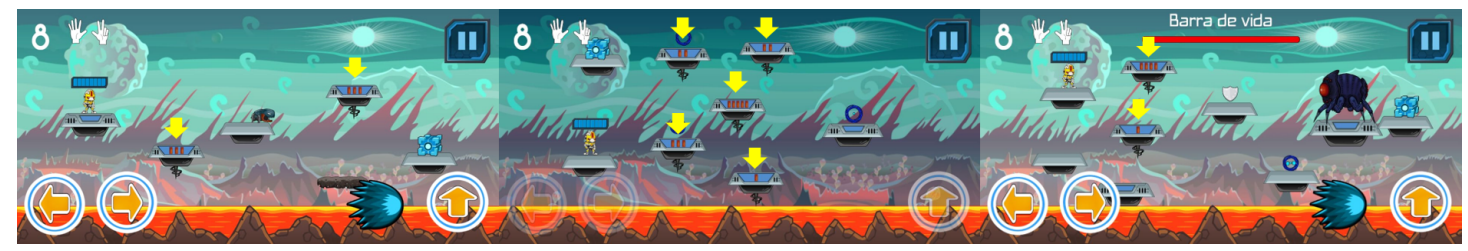

Figura 2. Mapeamento de plataformas. Na primeira tela existem duas plataformas consumidoras de energia, representadas por setas amarelas. Na segunda existem cinco. Na terceira, que é classificada como "desafio final", existem duas plataformas consumidoras de energia e o desafio com o boss.

Do primeiro ao último desafio, a utilização de elementos gráficos (plataformas sem e com indicadores de cargas de energia negativa; indicação de status de nível de energia, missões disponíveis; recursos a serem capturados; elementos que promovem $f e$ edback) auxilia a autoeficácia durante os desafios, indicando o progresso do jogador por toda a jornada do herói. 


\section{Materiais e Métodos}

Nesta seção são especificadas as características de implementação da pesquisa, os dados escolhidos para coleta, a forma de captura de dados e a proposta de análise. O fluxo de captura e análise foi estruturado conforme a Figura 3. De forma geral, os dados são capturados na Unity, enviados por e-mail para o Google Drive e analisados através do Python, especificamente com os recursos da biblioteca Pandas.

Coleta

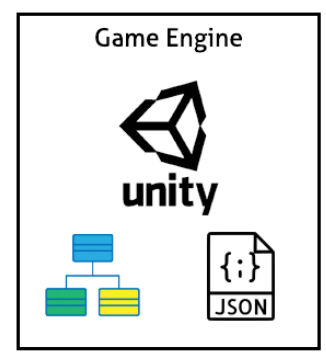

Armazenamento

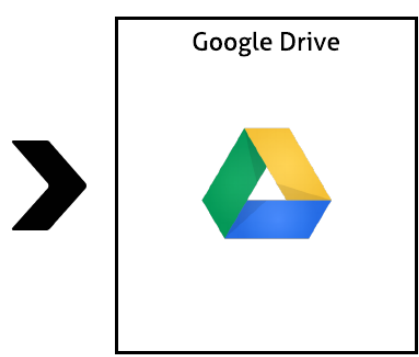

Análise

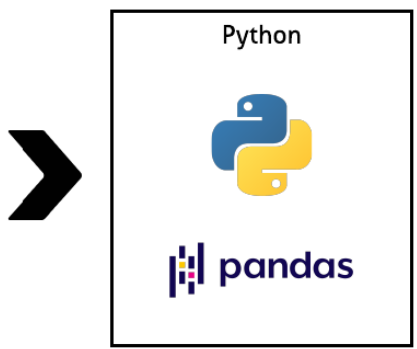

Figura 3. Fluxo de captura e análise de dados: os dados capturados na Unity são enviados por e-mail para o Google Drive para posterior análise através da biblioteca Pandas do Python.

\subsection{Dados Coletados}

Podem ser capturados diversos dados em um jogo, tanto de interface, quanto de mecânica, em alguns casos esses dados se relacionam, gerando uma informação relevante para análise. Dependendo do gênero e estilo de jogo, alguns dados são essenciais no momento da captura, pois esses podem representar o percurso do jogador com mais clareza. No jogo Robô Euroi, que possui gênero aventura puzzle em plataforma, foram armazenados os seguintes dados:

- Dados sociais: foi capturado o gênero e faixa etária do jogador, para no momento da análise, verificar se existem diferenças entre esses grupos;

- Data/hora de inicialização do jogo: dado capturado com o intuito de verificar o momento que o jogador inicializa o jogo;

- Tempo em jogo: cronômetro, que começa a contar desde o momento que o jogo foi inicializado, até o momento que o jogo é completado ou encerrado;

- Fases desbloqueadas: armazena a quantidade de fases que o jogador conseguiu desbloquear durante sua sessão;

- Dados da fase: tem como objetivo armazenar os valores de uma determinada fase do jogo, são esses: número da fase, status (fase bloqueada ou desbloqueada) e a quantidade de vezes que a fase foi jogada; e

- Dados das seções: podem ser geradas diversas seções dentro de uma fase, logo este dado tem como objetivo armazenar valores gerados em cada sessão, como: resultado da trajetória do jogador (vitória ou derrota), tempo da sessão em fase, percurso do jogador (lista de plataformas que ele pulou) e uma variável para armazenar o motivo da derrota, podendo ser mecânica, caso o usuário erre, por 
exemplo, o pulo de uma plataforma para outra (caindo na lava) ou lógica, caso pule em uma plataforma que retira mais energia do que ele tem atualmente.

\subsection{Estrutura e Organização}

Para realizar a captura de dados no jogo, foi desenvolvida uma estrutura própria, visto que a maioria dos modelos presentes na literatura são generalistas, implicando na perda de flexibilidade ao definir os dados, que variam de acordo com o jogo e podem ser específicos. Para modelar esta estrutura, foram utilizadas características de algumas ferramentas, como por exemplo o JSON (JavaScript Object Notation), que foi utilizado na Experience API [Kevan and Ryan 2016] e se mostrou eficaz no processo de armazenar valores de forma flexível.

A game engine utilizada para o desenvolvimento do jogo é o Unity 3D com a linguagem de programação C-Sharp, possibilitando a utilização de conceitos de POO (Programação Orientada a Objetos). Foram implementadas classes no C-Sharp que representassem a lista de dados (Seção 4.1) a ser coletada. Para melhor representação desta estrutura, foi desenhado o diagrama de classe, apresentado na Figura 4.

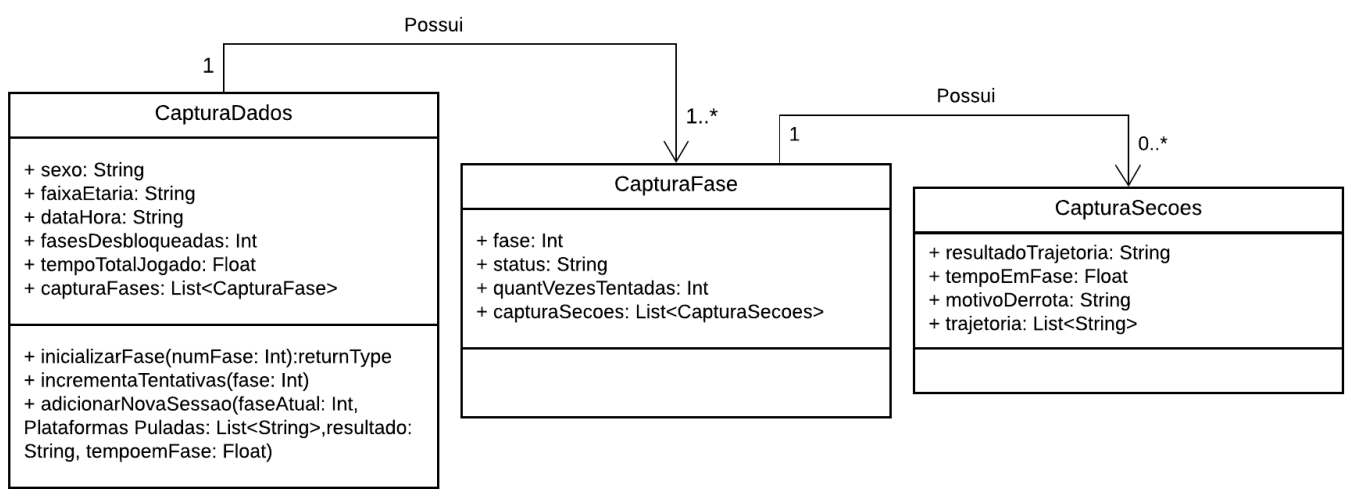

Figura 4. Diagrama de classes elaborado para representar o relacionamento das classes para armazenamento.

Após a finalização do diagrama e a implementação das classes na Unity, os campos dessas classes já estavam prontos para receber os seus respectivos registros, retirados do jogo em um determinado momento. Esta etapa foi desenvolvida para organizar e capturar os dados dentro da engine, não sendo possível enviá-los para outro local, pois estão em formato de classe.

Objetivando resolver o problema de exportação, foi utilizada uma técnica de "serialização", que permite transformar uma estrutura de dados ou um objeto em um formato que pode ser enviado e deserializado por diferentes aplicações. Neste trabalho, a estrutura foi serializada para o formato JSON, um formato de texto independente da linguagem, atualmente é reconhecido pela maioria das linguagens de programação. JSON é constituído pela estrutura de nomes/valores, similar a um dicionário /struct/array associativo, onde à esquerda fica chave e à direita o seu valor. Após o processo de serialização, o arquivo JSON é enviado para o Google Drive (armazenador) e deserializado utilizando a linguagem de programação Python. 
IX Congresso Brasileiro de Informática na Educação (CBIE 2020)

Anais do XXXI Simpósio Brasileiro de Informática na Educação (SBIE 2020)

\subsection{Proposta de Análise de Dados}

É possível gerar análises de vários objetos de aprendizagem, mas, quando este diagnóstico é realizado se baseando em elementos oriundos de um jogo digital, o número de possibilidades é maior do que muitas outras formas de conteúdo, pois em uma curta sessão, o jogador realiza diversas interações. Isso ocorre pela forma que alguns jogos são desenvolvidos, requerendo que a todo momento o usuário realize uma determinada ação [Freire et al. 2016]. Nesta pesquisa são executadas propostas de análises, com intuito de validar o que pode ser experimentado, utilizando os elementos descritos na seção 4.1. A Tabela 1 apresenta as análises realizadas e a descrição de cada uma.

Tabela 1. Tipos de análise possíveis de serem realizadas a partir dos dados coletados na utilização do jogo.

\begin{tabular}{|l|l|}
\hline Análise & Objetivo \\
\hline $\begin{array}{l}\text { Desempenho entre os gru- } \\
\text { pos }\end{array}$ & $\begin{array}{l}\text { Analisar a quantidade de tentativas realizadas pelo jogador em cada } \\
\text { fase, a fim de comparar o desempenho dos grupos (adultos, crianças) }\end{array}$ \\
\hline Level Design & $\begin{array}{l}\text { Verificar se a trajetória do jogador estava de acordo com o esperado } \\
\text { pelos desenvolvedores e validar a proposta de múltiplas soluções para } \\
\text { cada fase }\end{array}$ \\
\hline Tipos de derrota & $\begin{array}{l}\text { Verificar se a derrota foi causada por um erro de mecânica ou de lógica, } \\
\text { a fim de corrigir possíveis erros de modelagem nas fases }\end{array}$ \\
\hline Desistência & Analisar pontos no jogo que apresentaram o maior nível de desistência \\
\hline
\end{tabular}

\section{Resultados e discussão}

O jogo foi utilizado por 71 usuários, de três grupos distintos: crianças menores, públicoalvo e adultos, sendo doze crianças com idades menores que o público-alvo, 53 crianças pertencentes ao público-alvo e seis adultos. O tempo de seção para cada jogador foi limitado a 45 minutos, tempo médio suficiente para finalizar as treze fases ou desistir do jogo. Os experimentos com o público-alvo foram realizados em dois ambientes distintos: em casa e na sala de aula. As crianças menores jogaram na sala de aula, sendo disponibilizada ajuda, caso houvesse dúvidas. Além disso, foram capturados relatos, em áudio, com a percepção dos jogadores sobre o jogo.

O gráfico da Figura 5 apresenta a quantidade de tentativas em cada fase, a linha rosa refere-se às crianças menores, a vermelha ao público-alvo, a azul aos adultos e a linha verde é a média entre os jogadores. A partir desse gráfico, é possível visualizar que o grupo de crianças menores teve mais dificuldade nas fases iniciais, quando comparado aos outros grupos. A dificuldade sentida pelos menores pode ter influenciado na desistência, uma vez que, a partir da fase sete a maioria das crianças desistiu de jogar, justificando a diminuição no número de tentativas. O grupo dos adultos teve mais facilidade nas fases iniciais, se mantendo próximo ao público-alvo, que obteve resultados próximos à média.

Através do gráfico da Figura 5 também é possível visualizar pontos não previstos pelos projetistas do jogo, por exemplo, as fases dois e cinco tiveram um alto número de tentativas por parte das crianças menores, quase três vezes maior que a quantidade de tentativas da média geral, os motivos para isso podem ser a dificuldade de leitura ou dificuldade para realizar operações de soma e subtração. Com relação ao level design do jogo entre as fases um e doze, os resultados foram de acordo com o esperado (com exceção das crianças menores). A fase treze foi projetada para ser mais complexa, porém 


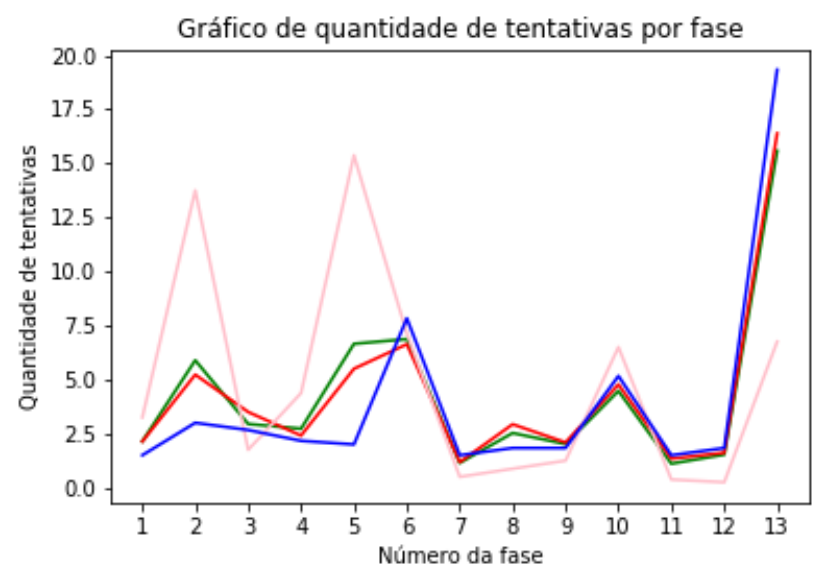

Figura 5. Número de tentativas por fase. Linha rosa, crianças com idade abaixo do público-alvo do jogo. Linha vermelha, público-alvo. Linha azul, adultos, com idade maior que as idades do público-alvo e linha verde, média das idades dos jogadores.

não esperava-se que o número de tentativas fosse tão alto. O possível motivo para isso é a apresentação de uma nova mecânica, onde o jogador se encontrava em um ambiente mais frenético, tendo menos tempo para pensar em suas ações. Um ponto positivo no alto número de tentativas, é o potencial do jogo em manter os jogadores motivados em alcançar o objetivo.

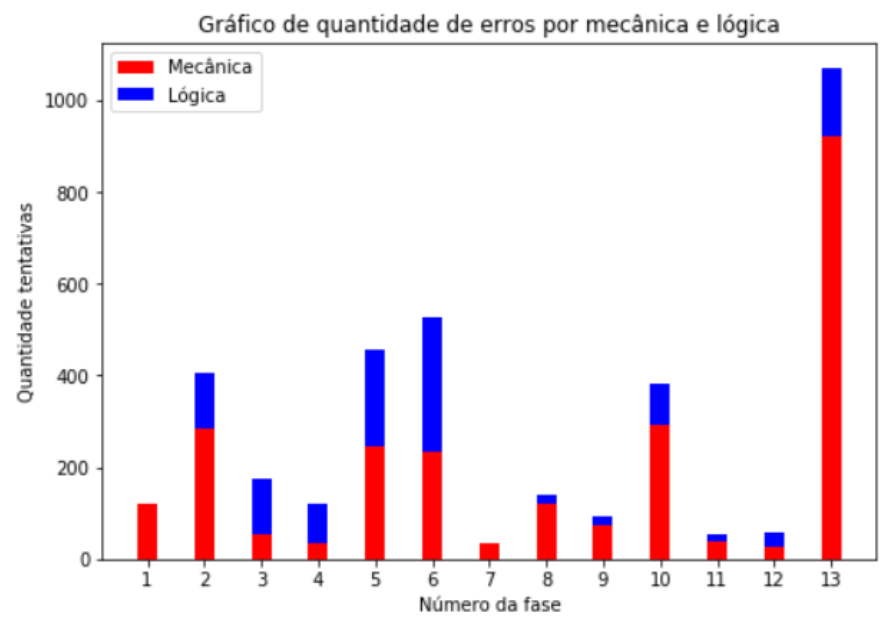

Figura 6. Comparação dos erros observados a partir dos dados. As barras vermelhas representam os erros de mecânica e as barras azuis representam os erros de lógica.

Uma das análises feitas foi a verificação do motivo de derrota, podendo ser por lógica (quando o jogador toma uma decisão errada, resultando em perda de mais energia do que poderia gastar) ou por mecânica (quando o jogador erra ao pular de uma plataforma para outra, caindo na lava). Como pode ser visto na Figura 6, a maioria dos erros foi de mecânica, o que pode indicar um erro na modelagem das fases ou que os jogadores não estão familiarizados com jogos do estilo plataforma, indicando a necessidade de incluir uma fase de aprendizagem (tutorial). As fases que tiveram mais erros de lógica do 
que mecânica apresentavam mais opções de caminhos a seguir, o que pode ter acarretado no erro. Foi observado que as três últimas fases apresentaram o maior número de desistências, provavelmente pelo fato de serem mais difíceis. Este fato é corroborado pelas percepções dos jogadores que comentaram nos áudios que o jogo mudava rapidamente de uma fase fácil para uma fase muito difícil.

\section{Conclusões e Trabalhos Futuros}

Este trabalho teve por objetivo analisar informações oriundas da utilização de um jogo educacional e propor uma estrutura flexível de captura de dados, contribuindo, de forma indireta, com o fomento de pesquisas na área de análise de aprendizagem em jogos educacionais no Brasil. Com os dados capturados a partir da utilização do jogo Robô Euroi, por 71 jogadores (crianças menores, público-alvo e adultos), foi possível realizar análises usando a biblioteca Pandas, da linguagem de programação Python. Neste trabalho foram realizadas análises sobre o desempenho entre os grupos, verificação do level design, motivo do erro do jogador e desistência do jogo.

A técnica de captura e análise utilizada permitiu otimizar a avaliação que geralmente é feita através de testes heurísticos e observações, facilitando tanto a aplicação para os estudantes, quanto a avaliação para os pesquisadores. $\mathrm{O}$ jogo apontou bons resultados com o público-alvo, e mostrou potencial para manter o jogador motivado em resolver os desafios, devido ao alto número de tentativas observado na análise. Alguns jogadores optaram por mandar um feedback sobre o jogo, evidenciando a importância de adicionar mais fases, melhorar o design, adicionar customização do personagem, disponibilizar o jogo em uma plataforma.

Embora o foco deste trabalho não tenha sido a avaliação da aprendizagem, os dados capturados e a estrutura proposta podem auxiliar no desenvolvimento de trabalhos nessa área. A análise de dados pode auxiliar na identificação de padrões de comportamento através de ações que podem permitir realizar avaliações de desempenho. Pois o ritmo de jogo tem relação com as estruturas de autorregulação da aprendizagem. Em jogos sérios, a mecânica deve estar ligada ao design de aprendizagem do jogo, para avaliá-la é necessário identificar as ações do jogador durante as fases. Um dos dados capturados neste trabalho foi a trajetória, que mostra qual caminho o jogador decidiu trilhar em cada fase, os elementos envolvidos nesses caminhos estão atrelados tanto à aprendizagem Matemática quanto a resolução de problemas de forma lógica. Cada jogo sério tem um objetivo e uma mecânica diferente, portanto, os dados a serem capturados devem ser modelados de maneira a compreender as estruturas de aprendizagem propostas.

Com os resultados apresentados neste trabalho, pretende-se fazer como trabalhos futuros, em dois âmbitos diferentes: (1) do jogo, melhorar o level design do jogo: adicionar mais fases, elementos e pontuação; (2) da análise: verificar os motivos que levaram o jogador a desistir, tornar a captura de dados mais implícita e fazer análises mais robustas.

\section{Referências}

Alonso-Fernández, C., Calvo-Morata, A., Freire, M., Martínez-Ortiz, I., and FernándezManjón, B. (2019a). Applications of data science to game learning analytics data: A systematic literature review. Computers \& Education, 141:103612. 
Alonso-Fernández, C., Cano, A. R., Calvo-Morata, A., Freire, M., Martínez-Ortiz, I., and Fernández-Manjón, B. (2019b). Lessons learned applying learning analytics to assess serious games. Computers in Human Behavior, 99:301-309.

Djaouti, D., Alvarez, J., Jessel, J.-P., and Rampnoux, O. (2011). Origins of serious games. In Serious games and edutainment applications, pages 25-43. Springer.

Freire, M., Serrano-Laguna, Á., Manero, B., Martínez-Ortiz, I., Moreno-Ger, P., and Fernández-Manjón, B. (2016). Game learning analytics: learning analytics for serious games. In Learning, design, and technology, pages 1-29. Springer Nature Switzerland AG.

Girard, C., Ecalle, J., and Magnan, A. (2013). Serious games as new educational tools: how effective are they? a meta-analysis of recent studies. Journal of computer assisted learning, 29(3):207-219.

Hicks, D., Eagle, M., Rowe, E., Asbell-Clarke, J., Edwards, T., and Barnes, T. (2016). Using game analytics to evaluate puzzle design and level progression in a serious game. In Proceedings of the Sixth International Conference on Learning Analytics \& Knowledge, pages 440-448.

Kang, J., Liu, M., and Qu, W. (2017). Using gameplay data to examine learning behavior patterns in a serious game. Computers in Human Behavior, 72:757-770.

Kevan, J. M. and Ryan, P. R. (2016). Experience api: Flexible, decentralized and activitycentric data collection. Technology, knowledge and learning, 21(1):143-149.

Melo, D., de Sousa Pires, F. G., Melo, R., and Júnior, R. J. d. R. S. (2018a). Robô euroi: Game de estratégia matemática para exercitar o pensamento computacional. In Brazilian Symposium on Computers in Education (Simpósio Brasileiro de Informática na Educação-SBIE), volume 29, page 685.

Melo, D., Júnior, R. J. d. R. S., Duarte, J., and de Sousa Pires, F. G. (2018b). Robô euroi: Estratégias matemáticas para desenvolver o pensamento computacional. In Anais dos Workshops do Congresso Brasileiro de Informática na Educação, volume 7, page 242.

Pires, F., Pessoa, M., Ferreira, R., Bernardo, J. R., and de Lima, F. M. (2020). O livro do conhecimento: um serious game educacional para aprendizagem de ortografia da língua portuguesa. Revista Brasileira de Informática na Educação, 28:436.

Plass, J. L., Homer, B. D., and Kinzer, C. K. (2015). Foundations of game-based learning. Educational Psychologist, 50(4):258-283.

Van Eck, R. (2006). Digital game-based learning: It's not just the digital natives who are restless. EDUCAUSE review, 41(2):16.

Wallner, G. and Kriglstein, S. (2013). Visualization-based analysis of gameplay data-a review of literature. Entertainment Computing, 4(3):143-155. 